\title{
PREVALENCE OF MICROALBUMINURIA WITH ISCHEMIC HEART DISEASE IN A SOUTH INDIAN POPULATION
}

\author{
Sheela Krishna Murthy'1, Shankar Donki², Balachandra $G^{3}$, Mithun Somaiah4, Jalaja $B^{5}$ \\ ${ }_{1}^{1}$ Assistant Professor, Department of General Medicine, BGS GIMS Hospital, Bangalore, Karnataka, India. \\ 2Professor and Unit Chief, Department of General Medicine, BGS GIMS Hospital, Bangalore, Karnataka, India. \\ 3 Professor, HOD and Unit Chief, Department of General Medicine, BGS GIMS Hospital, Bangalore, Karnataka, India. \\ ${ }^{4}$ Associate Professor, Department of General Medicine, BGS GIMS Hospital, Bangalore, Karnataka, India. \\ ${ }_{5}^{5}$ Assistant Professor, Department of General Medicine, BGS GIMS Hospital, Bangalore, Karnataka, India.
}

\section{ABSTRACT}

\section{BACKGROUND}

Microalbuminuria, or a moderate increase in the level of urine albumin, is now regarded as a risk factor for atherosclerosis. To reduce the burden of cardiovascular disease, management strategies are focusing more on preventive measures with early detection of markers of atherosclerosis. This study focuses on microalbuminuria, which is gaining recognition as a simple marker of atherosclerosis. Patients with microalbuminuria are at a greater risk for ischemic heart disease. An electrocardiogram, or ECG, can give information about heart disease, but reports relating ECG characteristics with and without microalbuminuria are lacking. In this clinical observational project, ischemic ECG findings of patients with microalbuminuria were studied. We wanted to determine the association between microalbuminuria and ischemic heart disease in a South Indian population.

\section{METHODS}

A cross-sectional study was conducted within a cohort of 202 patients, aged 40 years or older. All patients underwent routine blood investigations, urine analysis examination and a resting 12-lead ECG and were screened for microalbuminuria and risk factors for coronary artery disease as well as ischemic ECG abnormalities.

\section{RESULTS}

Conduction abnormalities were less commonly seen in patients with some amount of proteinuria (8\%). ECG abnormalities, especially left ventricular hypertrophy +/- strain pattern, were seen in about one-third patients with proteinuria (35\%). ST segment changes (elevation or depressions) were seen more in patients with co-existing comorbidities, like Type 2 diabetes mellitus or obesity.

\section{CONCLUSIONS}

This study shows the importance of early detection of microalbuminuria and screening for asymptomatic coexisting cardiac disorders. The purpose of this study was to test the hypothesis that identification of microalbuminuria leads to further evaluation of coronary artery disease regardless of other comorbid conditions.

\section{KEY WORDS}

Microalbuminuria, CAD, ECG, IHD, Proteinuria, Atherosclerosis

HOW TO CITE THIS ARTICLE: Murthy SK, Donki S, Balachandra G, et al. Prevalence of microalbuminuria with ischemic heart disease in a South Indian population. J. Evolution Med. Dent. Sci. 2019;8(23):1834-1838, D0I: 10.14260/jemds/2019/403

\section{BACKGROUND}

Microalbuminuria, or a moderate increase in the level of urine albumin, is now regarded as a risk factor for atherosclerosis. [1] To reduce the burden of cardiovascular disease, management strategies are focusing more on preventive measures with early detection of markers of atherosclerosis. This study focuses on microalbuminuria, which is gaining recognition as a simple marker of atherosclerosis.

'Financial or Other Competing Interest': None.

Submission 07-12-2018, Peer Review 24-05-2019,

Acceptance 31-05-2019, Published 10-06-2019.

Corresponding Author:

Dr. Sheela Krishna Murthy,

No. A-104,

Gagan Castle Apartment,

Rajarajeshwari Nagar,

Bangalore-560098,

Karnataka, India.

E-mail: sheelakm@gmail.com

DOI: $10.14260 /$ jemds $/ 2019 / 403$
Prospective and epidemiologic studies have found that microalbuminuria is predictive, independently of traditional risk factors, of all-cause and cardiovascular mortality and CVD events within groups of patients with diabetes or hypertension, and in the general population. Microalbuminuria can infer the risk of cardiovascular disease in diabetic patients, in elderly patients, and also in the general population. Although microalbuminuria is thought to be associated with a certain amount of sub-clinical atherosclerosis, it is still unknown how prematurely microalbuminuria appears in the atherosclerotic process. Global population studies show a relationship between microalbuminuria and insulin resistance, dyslipidaemia, increased BMI, and salt sensitivity in patients with Type 2 diabetes mellitus and systemic hypertension. Patients with microalbuminuria are also found to have an increased occurrence of retinal microvascular lesions and left ventricular hypertrophy.

Coronary artery disease has become one of the leading causes of mortality worldwide, nearly estimating more than 7.3 million deaths in 2008 alone. Additionally, more than $75 \%$ of these cardiac related mortalities occur from low- and 
middle-income countries. Over $25 \%$ of these occur in South Asia, therefore cardiovascular disease is responsible for $24 \%$ of all deaths in India. 31.8 million Indians have risk factors for ischemic heart disease and/or subclinical or asymptomatic heart disease. These shocking numbers, as well as the huge economic burden imposed by the morbidity and mortality associated with ischemic heart disease underscore the need for risk factor screening and early diagnosis in the general population. Many biomarkers have been proposed and evaluated including markers of systemic inflammation like Creactive protein and lipoprotein associated phospholipase A2 and indicators of subclinical atherosclerotic disease such as intima-media thickness and ankle-brachial index.

Microalbuminuria in diabetes mellitus is recognized as a marker for systemic atherosclerosis. Microalbuminuria has been defined as a urinary albumin excretion rate greater than $30 \mathrm{mg} / 24$ hours or a urine albumin creatinine ratio greater than $30 \mathrm{mg} / \mathrm{g}$ in an early morning sample. These cut-off values have been primarily defined for proteinuria in diabetic individuals and are yet to be rigorously validated in nondiabetic individuals. In terms of natural course of the disease in association with biomarkers and evolving ischemic heart disease, microalbuminuria occupies the early end of the spectrum, as an indicator of subclinical disease. The close association between microalbuminuria and coronary artery disease is readily explained by the shared pathogenesis of endothelial dysfunction, systemic inflammation and vascular injury. It is reasonable to assume that such a relationship should exist regardless of the concomitant presence or absence of diabetes.

The underlying mechanisms between microalbuminuria and the risk for cardiovascular disease are not completely understood; one explanation is that it may be due to more vascular leakiness of albumin in both renal and systemic vessels. It is recognized that microalbuminuria is an indicator of widespread endothelial dysfunction, and this may lead to an atherogenic state. Endothelial dysfunction may promote an increase in the permeation of lipoproteins and atherosclerosis in the arterial wall. However, insulin insensitivity, glycaemic status, a highly coagulable state, and adhesion molecules have all been presumed to be involved in the pathogenesis. Data recommends that in diabetic patients with hypertension, tight blood pressure control may lower the risk for proteinuria and that inhibition of the reninangiotensin-aldosterone system (RAAS) can delay or prevent the evolution of microalbuminuria to overt nephropathy in normotensive persons. ACE inhibitors have been accepted as first-line antihypertensive therapy in diabetic patients with proteinuria, and these drugs give unique support in slowing the progression of diabetic nephropathy and atherosclerosis. Whether albuminuria is a risk factor or just a marker for cardiovascular disease, it identifies those high-risk diabetic patients who should be treated for early and aggressive intervention. If microalbuminuria is persistent, blood pressure control should be combined with RAS inhibition to try stabilizing and reducing microalbuminuria, preserving kidney function and possibly lowering cardiovascular risk.

\section{Aims \& Objectives}

To determine an association between microalbuminuria and ischemic heart disease in a South Indian population

\section{METHODS}

\section{Study Design}

This was a Cross-sectional study.

\section{Study Place}

Study was planned and executed by the Department of General Medicine, BGS Global institute of medical sciences and Hospital, Bangalore, Karnataka during September 2017 to September 2018.

\section{Study Population}

Patients with age $\geq 40$ years, admitted to the Department of General Medicine in BGS GIMS Hospital, Bangalore. All patients underwent routine blood investigations, urine analysis examination and a resting 12-lead ECG and were followed for microalbuminuria and risk factors for coronary artery disease as well as electrocardiographic evidence of myocardial ischemia.

The only exclusion criterion was chronic kidney disease.

\section{Sampling Technique}

The universal sampling technique was used for all the patients matching the inclusion and exclusion criteria.

\section{Sample Size}

A 95\%-degree confidence corresponds to $\alpha=0.05$. Each of the shaded tails in the following figure has an area of $\% / 2=$ 0.025 . The region to the left of $z / 2$ and to the right of $z=0$ is $0.5-0.025$, or 0.475 . In the table of the standard normal ( $z$ ) distribution, an area of 0.475 corresponds to a $Z$ value of 1.96 . The critical value is therefore $z_{x / 2}=1.96$.

The margin of error $E=1$ and the standard deviation $\sigma=$ 6.95. Using the formula for sample size, we can calculate $n$ : $n=\left[\frac{z_{x / 2}{ }^{\sigma}}{\mathrm{E}}\right]^{2}=\left[\frac{1.96 \cdot 6.95}{1}\right]^{2}=[13.62]^{2}=185.55=186$

Therefore, we will need to sample at least 186 randomly selected patients. Here we have taken 202. With this sample we will be 95 percent confident that the sample mean $\bar{x}$ will be included.

\section{Data Analysis \& Statistics}

The data were entered in Microsoft Excel 2010 and analysed using SPSS software V 20.

Descriptive statistics was used, and results were expressed as Odds ratio and P-value. The statistical test used to find the P-value from odds ratio was the Fisher's exact test. Odds ratio is the statistic used to quantify the strength of the association between variables.

\section{RESULTS}

A total 202 patients were studied. Left ventricular hypertrophy with strain pattern, Q waves, QS complexes, non-progression of the $\mathrm{R}$ wave, ST segment elevation or depression, T-wave inversions, and left bundle branch block were considered ischemic ECG changes. Fifty-seven patients 
had ischemic ECG changes. The statistical method used was the inferential method.

This study attempts to find and association between microalbuminuria and ischemic heart disease.

The prevalence of ischemic ECG abnormalities, in general, increased with an increase in the amount of proteinuria.

Different mechanisms have been proposed to explain why patients with proteinuria have a higher risk of cardiovascular events. One potential mechanism may relate to systemic inflammation. Epidemiologic data strongly associates systemic inflammation to atherosclerosis and ischemic heart disease. ${ }^{[2]}$

\begin{tabular}{|c|c|c|c|c|c|c|c|}
\hline 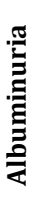 & 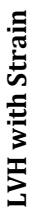 & $\sum_{0}^{\infty}$ & 己 & 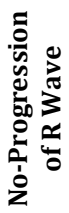 & 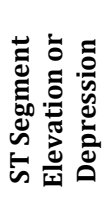 & 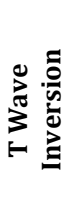 & $\stackrel{m}{m}$ \\
\hline $1+$ & 3 & 1 & 0 & 1 & 5 & 3 & 1 \\
\hline $2+$ & 1 & 2 & 1 & 2 & 4 & 3 & 0 \\
\hline $3+$ & 0 & 1 & 3 & 1 & 3 & 4 & 1 \\
\hline $4+$ & & 3 & 0 & 2 & 4 & 6 & 2 \\
\hline
\end{tabular}

Table 1. Ischemic ECG Changes Seen in Varying Degrees of Albuminuria

\begin{tabular}{|c|c|c|}
\hline Variable & Odds Ratio (95\% CI) & p-Value \\
\hline BMI & $0.96(0.70,1.32)$ & 0.80 \\
\hline Waist-Hip Ratio ${ }^{a}$ & $1.67(1.34,2.07)$ & $<0.001$ \\
\hline Systolic BP & $1.20(1.09,1.32)$ & $<0.001$ \\
\hline Diastolic BP & $1.18(1.04,1.35)$ & 0.01 \\
\hline Dyslipidaemia & $1.71(1.21,2.41)$ & 0.002 \\
\hline Smoking (Pack Years) & 1 & \\
\hline & $1.23(0.71,1.82)$ & 0.001 \\
\hline & $2.23(1.45,3.44)$ & \\
\hline Microalbuminuria & $1.04(0.67,1.62)$ & 0.84 \\
\hline \multicolumn{3}{|c|}{ Table 2. Risk Factors for Ischemic Heart Disease } \\
\hline adds ratios are giver & -unit increase in the & ory variab \\
\hline
\end{tabular}

\section{DISCUSSION}

Comorbid conditions like diabetes mellitus, chronic kidney disease and cardiovascular disease are strong predictors of mortality.[3] Early asymptomatic stages of CKD have been associated with moderate to severe cardiovascular disease. Dipstick positive proteinuria, albumin-creatinine ratio (ACR) and a reduced eGFR have all been linked with cardiovascular and all-cause mortality.[4] Hemmelgarn et al, a Canadian study, showed that gross proteinuria autonomously increased the progression of CKD, myocardial infarction (MI) and risk of death in certain patient groups. Data suggests that microalbuminuria has consequences for all-cause mortality and cardiovascular outcomes even in individuals with chronic kidney disease and not at only the general population level.[5] Population-based cohort studies reveal that multivariable relative risks of cardiovascular disease mortality for proteinuria range from 1.2-2.9.[6] In terms of cardiovascular morbidity, albumin-creatinine ratio and dipstick-positive proteinuria are found to be prognosticators of cardiovascular diseases including stroke, coronary artery disease, and hypertension in the general population, with some studies proposing that proteinuria is a stronger predictor of outcome than the traditional risk factors, such as blood pressure and cholesterol.[7] The Heart Outcomes Prevention Evaluation (HOPE) study revealed that proteinuria was associated with negative outcomes independent of conventional cardiovascular risk factors. ${ }^{[8]}$ Moreover, the atherosclerosis risk appears to be proportional to the levels of urinary protein excretion, even if not pathological. In patients with hypertension, studies reveal that microalbuminuria produces a 4 times bigger risk for consequent coronary artery disease than in patients without albuminuria.[9] This effect appears to be independent even of the well-known risk factors for atherosclerosis. Furthermore, the presence of left ventricular hypertrophy in hypertensive or diabetic patients has been associated with albuminuria. It has also been shown that proteinuria confers increased risks of cardiovascular as well as all-cause and mortality, even at a microalbuminuria level in individuals with stable underlying ischemic heart disease.[10] The same has been identified in those patients who have recently suffered a coronary event. Macroalbuminuria, not microalbuminuria, was shown to be a better predictor of mortality in the Pravastatin or Atorvastatin Evaluation and Infection Therapy-Thrombolysis in Myocardial Infarction (PROVE IT-TIMI 22) study. The Strong Heart Study strongly established the association between albuminuria and abnormal systolic and diastolic ventricular function in diabetic patients.[11] A decrease in adiponectin levels and elevated C-reactive protein (CRP) is shown to be associated with microalbuminuria; a significant relationship is also seen between the degree of proteinuria and CRP levels. Evidence also relates asymmetric dimethylarginine (ADMA) with proteinuria. ADMA is a recognized biomarker of inflammation causing endothelial dysfunction by inhibiting the production of nitric oxide. [12] Von Willebrand Factor (vWF) antigen is released in larger quantity as a response to endothelial cell damage. In addition, thrombogenic factors have been linked as probable explanations in the mechanisms between proteinuria and ischemic heart disease. Fibrinogen and tissue plasminogen activator are also evident in urinary albumin excretion. It suggests that an environment with high platelet aggregation and adhesiveness seen in patients with proteinuria could lead to an increased risk of thrombosis. Most of the studies that try to hypothesize a relationship between proteinuria and cardiovascular disease are cross-sectional and so any conclusions regarding mortality cannot be made.

Proteinuria appears to be associated with a greater risk of cardiovascular morbidity and mortality and is emerging as a screening tool for cardiovascular risk reduction. The RENAAL study revealed a lower cardiovascular event rate was seen is patients with bigger reductions in the urinary albumin excretion. Analogous findings were published from the LIFE study. Current guidelines focus on the significance of RAAS inhibition as first line therapy in terms of slowing down the progression of nephropathy and reducing proteinuria in these patients, with additional antihypertensive medications alongside ACEIs or ARBs for adequate control of blood pressure. Cardiovascular risk management in patients with proteinuria aims at reducing the degree of proteinuria along with modification of other risk factors like blood pressure, insulin resistance, dyslipidaemia, smoking, and obesity.[13]

Strategies to reduce microalbuminuria are available in clinical practice, most important is the reduction of blood pressure. In the Irbesartan Microalbuminuria-2 trial, reducing microalbuminuria in patients with type 2 diabetes and hypertension occurred in approximately $20 \%$ of patients 
who attained traditional BP goals of 140/90 mmHg with medications such as diuretics, $\beta$ blockers, and vasodilators. An improvement in attaining target blood pressure to approximately $33 \%$ occurred when the full dosage (300 mg) of the renin-angiotensin system blocking drug irbesartan (An angiotensin II receptor blocker) was used as part of the BPlowering regimen to $140 / 90 \mathrm{mmHg}$. However, even lower BP goals may be advantageous for reducing microalbuminuria, and both the Joint National Committee 7 and the ADA recommend a lower BP goal of $<130 / 80 \mathrm{mmHg}$ in patients with diabetes. Higher dosage angiotensin receptor blocker or using both an ACE inhibitor and an angiotensin receptor blocker together in full dosage may facilitate an even greater reduction in microalbuminuria. Thiazide diuretics also help to reduce proteinuria, and dietary salt restriction may also be helpful in reducing microalbuminuria. Strict glycaemic control also delays the onset of microalbuminuria, the progression of microalbuminuria to clinical proteinuria, and the development of nephropathy in patients with either type 1 or type 2 diabetes.[14] The Steno- 2 trial demonstrated that intensified BP, cholesterol, and glycaemic control in patients with type 2 diabetes was associated with decreased risk for cardiovascular events.[9] However, this was not correlated with reduction in microalbuminuria. There is some debate in the literature as to whether statins reduce microalbuminuria. Regardless, these drugs are important given the CVD risk. Both the National Kidney Foundation and the ADA recommend an LDL cholesterol goal of $<100 \mathrm{mg} / \mathrm{dl}$ for patients with advanced renal disease or diabetes. For patients with diabetes and CVD, an LDL goal of $<70 \mathrm{mg} / \mathrm{dl}$ is appropriate. Abnormalities in triglyceride, HDL, and non-HDL levels should also be highlighted and treated. Moreover, one needs to consider strategies to improve diet by decreasing the intake of saturated, transfats and dietary salt. In addition, efforts to assist in smoking avoidance, proper exercise, and weight control should be encouraged. Annual screening for microalbuminuria is recommended in people with diabetes. In part, this recommendation is based on the evidence that long-term cost-effectiveness is more favourable when treatment is started earlier in preventing ESRD, but there are no data when it comes to preventing CVD. The evidence for screening the general population without diabetes for microalbuminuria to prevent end-stage renal disease is limited.[15] Only in the past year was a study that evaluated the cost-effectiveness of screening for microalbuminuria in the general population published. In this analysis, patients with increased microalbuminuria were treated with an ACE inhibitor. The authors demonstrated that this approach was cost-effective to prevent cardiovascular events. Although evidence is accumulating to indicate that individuals with hypertension and increased cardiovascular risk should be screened for microalbuminuria, more studies are needed to confirm that systematic screening is cost-effective in the general population.

\section{CONCLUSIONS}

Clinical evidence has established a pathophysiologic connection between microalbuminuria and coronary artery disease in patients with diabetes and hypertension and in the general population. This correlation is observed even at levels of albuminuria below the conventional threshold for microalbuminuria. Screening for microalbuminuria can help clinicians estimate a patient's cardiac risk and, if positive, should prompt the early introduction of a reduction in risk factors to improve the overall cardiovascular risk factor profile as well as prevent further loss of renal function. This population-based study demonstrates that microalbuminuria is a clinically relevant risk marker for the development of ischemic heart disease. Microalbuminuria predicts coronary artery disease independently of other classic atherosclerotic risk factors and moreover, relates with the effect of smoking, suggesting that there is an increased atherogenic susceptibility. Thus, individuals with other atherosclerotic risk factors such as smoking, diabetes, hypertension and dyslipidaemia should have their microalbuminuria measured, which gives information contributing to the classification of the individual as a high-risk or highly-susceptible individual.[16] It is unknown whether individuals with microalbuminuria will benefit more from intervention, but it leads to a more targeted and focused strategy for the prevention of ischemic heart disease.

\section{REFERENCES}

[1] De Zeeuw D, Parving $\mathrm{HH}$, Henning RH. Microalbuminuria as an early marker for cardiovascular disease. J Am Soc Nephrol 2006;17(8):2100-5.

[2] Smith JW, Marcus FI, Serokman R, et al. Prognosis of patients with diabetes mellitus after acute myocardial infarction. Am J Cardiol 1984;54(7):718-21.

[3] Kannel WB, McGee DL. Diabetes and cardiovascular disease. The Framingham Study. JAMA 1979;241(19):2035-8.

[4] Ljungman S, Wikstrand J, Hartford M, et al. Urinary albumin excretion - a predictor of risk of cardiovascular disease. A prospective 10-year followup of middle-aged non-diabetic normal and hypertensive men. Am J Hypertens 1996;9(8):770-8.

[5] Garg AX, Kiberd BA, Clark WF, et al. Albuminuria and renal insufficiency prevalence guides population screening: results from the NHANES III. Kidney Int 2002;61(6):2165-75.

[6] Yoshino H, Matsuoka K, Nishimura F, et al. Painless myocardial infarction in diabetics. Tohoku J Exp Med 1983;141 Suppl:547-54.

[7] Sarnak MJ, Levey AS, Schoolwerth AC, et al. Kidney disease as a risk factor for development of cardiovascular disease: a statement from the American Heart Association Councils on Kidney in Cardiovascular Disease, High Blood Pressure Research, Clinical Cardiology and Epidemiology and Prevention. Circulation 2003;108(17):2154-69.

[8] Agrawal V, Marinescu V, Agarwal M, et al. Cardiovascular implications of proteinuria: an indicator of chronic kidney disease. Nat Rev Cardiol 2009;6(4):301-11.

[9] Kashif W, Siddiqi N, Dincer AP, et al. Proteinuria: how to evaluate an important finding. Cleve Clin J Med 2003;70(6):535-7, 541-4, 546-7. 
[10] Culleton BF, Larson MG, Parfrey PS, et al. Proteinuria as a risk factor for cardiovascular disease and mortality in older people: a prospective study. Am J Med 2000;109(1):1-8.

[11] Segura J, Campo C, Ruilope LM. Proteinuria: an underappreciated risk factor in cardiovascular disease. Curr Cardiol Rep 2002;4(6):458-62.

[12] Wagner DK, Harris T, Madans JH. Proteinuria as a biomarker: risk of subsequent morbidity and mortality. Environ Res 1994;66(2):160-72.

[13] Garcia MJ, McNamara PM, Gordon T, et al. Morbidity and mortality in diabetics in Framingham population. Sixteen year follow-up study. Diabetes 1974;23(2):105-11.
[14] Rubler S, Gerber D, Reitano J, et al. Predictive value of clinical and exercise variables for detection of coronary artery disease in men with diabetes mellitus. Am J Cardiol 1987;59(15):1310-13.

[15] Jarrett RJ, Viberti GC, Argyropoulos A, et al. Microalbuminuria predicts mortality in non-insulindependent diabetes. Diabetic Med 1984;1(1):17-9.

[16] Mogensen CE. Microalbuminuria predicts clinical proteinuria and early mortality in maturity-onset diabetes. N Engl J Med 1984;310(6):356-60. 